\title{
Diagnosis and Management of Hypermobility Spectrum Disorders in Primary Care
}

\author{
Karina Atwell, MD, MPH, William Michael, MD, Jared Dubey, DO, Sarah James, DO, \\ Andrea Martonffy, MD, Scott Anderson, MS, Nathan Rudin, MD, and \\ Sarina Schrager, $M D$, MS
}

Hypermobility spectrum disorders (HSDs) encompass an array of connective tissue disorders characterized by joint instability and chronic pain. Fatigue and other systemic symptoms that affect daily functioning may occur, as well. Accurate data on incidence and prevalence of HSDs is hampered by lack of awareness of these conditions and the wide heterogeneity of their clinical presentation. Identifying which type of HSD is present is important in guiding appropriate care. In particular, making the diagnosis of hypermobile Ehlers-Danlos syndrome (hEDS) is important, as individuals with hEDS may be at risk for more significant multisystem involvement. Diagnostic criteria for hEDS include measures of joint hypermobility, skin and other connective tissue findings, and lack of evidence of a different type of Ehlers-Danlos syndrome. Beyond accurate diagnosis, HSDs pose many challenges for primary care providers, as ongoing patient education, patient empowerment, and coordination of a multidisciplinary treatment team are integral to proper care. This article describes the incidence and prevalence, pathophysiology, diagnosis, and management of HSDs, including clinical cases exemplifying how joint hypermobility might present within a primary care setting. (J Am Board Fam Med 2021;34:838-848.)

Keywords: Chronic Pain, Joint Hypermobility, Musculoskeletal System

\section{Case 1}

Jennifer is a 15 -year-old female who comes in with her mother to discuss her diffuse muscle and joint pain and "feeling tired all the time." She states that the pain is most intense in her shoulders, back, feet, and ankles. The pain has been present since "at least middle school" and has increased since starting high school. She has a history of recurrent shoulder dislocations starting at age 13. She also describes 1 episode of a patellar dislocation with normal $\mathrm{x}$-rays.

This article was externally peer reviewed.

Submitted 21 July 2020; revised 17 November 2020; accepted 17 November 2020.

From Department of Family Medicine and Community Health, University of Wisconsin School of Medicine and Public Health, Madison (KA, WM, JD, SJ, AM, SS); University of Wisconsin Department of Kinesiology, Madison (SA); Department of Orthopedics and Rehabilitation Medicine, University of Wisconsin School of Medicine and Public Health, Madison (NR).

Funding: None.

Conflict of interest: None.

Corresponding author: Sarina Schrager, MD, MS, Department of Family Medicine and Community Health, University of Wisconsin School of Medicine and Public Health, 1100 Delaplaine Ct., Madison, WI 53715 (E-mail: sbschrag@wisc.edu).
She describes herself as "double jointed." She has also sprained her ankles multiple times.

She gets 8 to 10 hours of restful sleep every night but still is barely able to make it through the school day without falling asleep, much less get home and do her homework. Her past medical history includes anxiety, chronic headaches, gastroesophageal reflux disease, irritable bowel syndrome (IBS), and orthostatic hypotension. She feels lightheaded when getting up quickly and has had an electrocardiogram that was normal. She has had an umbilical hernia repair as well as a revision. Her mother also has joint laxity, anxiety, and IBS.

Her vitals are normal. She can extend both elbows and knees to greater than 10 degrees, extend fifth metacarpophalangeal (MCP) joints past 90 degrees, and easily put the palms of her hands on the floor without bending her knees (Beighton score 7/9). The scar on her abdomen seems atrophic. Her skin is soft and mildly hyperextensible. There is livedo reticularis (skin translucency revealing subcutaneous veins). She demonstrates arachnodactyly, with thumbs and fifth fingers able 
to meet around the contralateral wrist (Walker wrist sign). There are pale stretch marks at the lateral hips, breasts, and axillae; she reports these developed during her adolescent growth spurt.

\section{Case 2}

Sonja is a 23-year-old woman who presents with widespread pain, headaches, insomnia, and anxiety, which began 18 months ago after a motor vehicle accident (MVA). Before the accident she had been in very good health, enjoyed long-distance running, worked at a coffee shop, and was enrolled in undergraduate studies. She did have some preaccident wrist, hand, and elbow pain associated with computer use. She suffered no major injuries in the MVA aside from whiplash, but months after the accident was still having significant neck pain and headaches as well as a range of new and disconcerting symptoms such as upper back pain, foot pain, tinnitus, chest pain, photophobia, and phonophobia.

Since that time, she had been referred to and evaluated by specialists in multiple disciplines including neurology, rheumatology, physical therapy, and sports medicine. She also had a variety of laboratory tests and imaging studies, all of which were normal. She had tried numerous medications including duloxetine, amitriptyline, naproxen, acetaminophen, and gabapentin. However, her symptoms worsened, and she began to experience significant fatigue. She started working with chiropractors, getting adjustments several times per week to "keep everything in place." The adjustments gave partial relief, but only for a day or 2 .

She left her job and took a leave of absence from school after the MVA and has been unable to return. She moved back in with her parents and spends much of the day sedentary, aside from short walks that leave her feeling achy and fatigued. She does not use alcohol, tobacco or other forms of nicotine, or illicit drugs.

She has no known family history of joint hypermobility.
On examination, she is somewhat anxious and expresses concern over her vertebrae being "out of place," as communicated by her chiropractor. She has a body-mass index of 20 and resting tachycardia at 105 beats per minute; other vital signs are normal. She has difficulty tracking during testing of extraocular movements, citing eye fatigue, but has no nystagmus. She has pain with neck motion in all planes. She has normal spinal range of motion but experiences spine pain with thoracic and lumbar flexion and side bending. Effort during manual muscle testing is limited due to pain, but she is able to mount grade 5 (normal) effort on repeated testing. Her Beighton score is 4/9, with points for fifth finger MCP hyperextension and elbow hyperextension bilaterally. Her skin is of normal softness and elasticity. There is no arachnodactyly.

\section{Joint Hypermobility and Hypermobility Spectrum Disorders-Definitions}

Joint hypermobility is a common complaint seen in primary care. Joint hypermobility refers to increased active or passive movement of a joint beyond its normal range. One can have joint hypermobility without having a hypermobility spectrum disorder (HSD). Joint hypermobility can be categorized by question-based and physical examinationbased measures. On a validated, commonly used patient questionnaire eliciting hypermobility symptoms, an affirmative answer to 2 or more out of 5 questions shows $84 \%$ sensitivity and $80 \%$ specificity for joint hypermobility (Table 1). The physical examination-based counterpart to this questionnaire is the Beighton hypermobility score (Table 2), which is commonly employed in the assessment of the hypermobile patient. ${ }^{3}$ Though the Beighton hypermobility scoring rubric was initially introduced as an instrument for epidemiologic research rather than a tool to be used diagnostically, it has now come to be used as a measure of generalized hypermobility. ${ }^{2}$ Higher scores on the Beighton

\section{Table 1. Validated Questionnaire for Joint Hypermobility}

1. Can you now (or could you ever) place your hands flat on the floor without bending your knees?

2. Can you now (or could you ever) bend your thumb to touch your forearm?

3. As a child, did you amuse your friend by contorting your body into strange shapes or could you do the splits?

4. As a child or teenager, did your kneecap or shoulder dislocate on more than one occasion?

5. Do you consider yourself double jointed?

Adapted from Hakim and Grahame. R. ${ }^{1}$ 
Table 2. Beighton Hypermobility Score-Maximum Score $=9$ Points

Can place hands flat on the floor without bending the knees

1 point

\begin{tabular}{lc} 
& 1 point \\
\hline Right & Left \\
1 point & 1 point \\
1 point & 1 point \\
1 point & 1 point \\
1 point & 1 point
\end{tabular}

Can passively dorsiflex the fifth metacarpophalangeal joint to $90^{\circ}$ or greater

Can oppose the thumb to volar aspect of forearm

Can hyperextend the elbow $10^{\circ}$ or more

Can hyperextend the knee $10^{\circ}$ or more

Adapted from Fikree et al. ${ }^{2}$

scale simply represent a greater number of affected joints rather than a higher degree of laxity.

\section{Hypermobility Spectrum Disorder- Diagnostic Criteria}

Kirk and colleagues first described hypermobility syndrome in 1967, describing a syndrome of familial ligamentous laxity resulting in recurrent joint pain and periodic joint effusion. ${ }^{4}$ It was thought to be isolated to the musculoskeletal system, separate from Marfan syndrome and the Ehlers-Danlos syndromes (EDSs), and to occur in otherwise healthy people. Over time, overlap in signs and symptoms has been noted with other hereditary disorders of connective tissue. There was considerable overlap between hypermobility syndrome and hypermobility-type (type III) EDS (hEDS), to the point that differentiating the 2 entities was very difficult (Figure 1).

From 2000 until 2017, patients with hypermobility were diagnosed with what was then called

Figure 1. Diagnosing disorders of joint hypermobility: distinguishing hypermobile Ehlers-Danlos syndrome and the hypermobility spectrum disorders. Abbreviations: hEDS, hypermobile Ehlers-Danlos syndrome; EDS, EhlersDanlos syndrome; HSD, Hypermobility spectrum disorder.

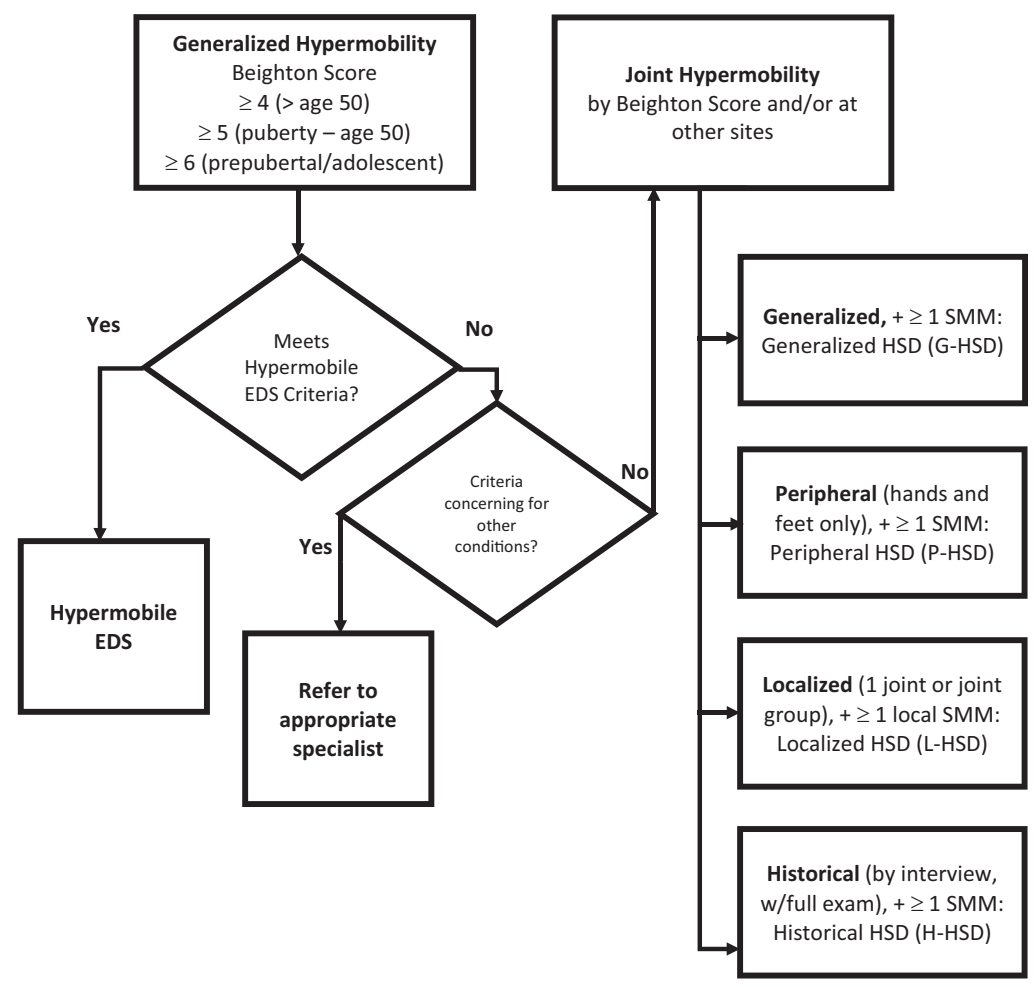


benign joint hypermobility syndrome (JHS), once the presence of another connective tissue disorder had been excluded. In 2017, JHS was reclassified and renamed the hypermobility spectrum disorders, ${ }^{5}$ which are subcategorized into generalized (G-HSD), peripheral (P-HSD), localized (L-HSD), and historic (H-HSD) types. At the same time, type
III EDS was renamed hypermobile EDS (hEDS) and given a new set of diagnostic criteria that better differentiate it from G-HSD (Table 3). If significant hypermobility is present on the Beighton hypermobility score, but a patient does not fit the criteria for hEDS or another of the EDSs, the patient is diagnosed as G-HSD. Yet, the 2 disorders

Table 3. Diagnostic Criteria for Hypermobile Ehlers-Danlos Syndrome (hEDS) ${ }^{3}$

Criterion 1-generalized joint hypermobility

Beighton score _ 19

$\cdot \geq 6$ for prepubertal children and adolescents

$\cdot \geq 5$ for pubertal men and woman to age 50

.$\geq 4$ for men and women over age 50

If Beighton score is 1 point below age-specific cutoff, affirmative answer to 2 of the following questions will meet criterion 1.

- Can you now (or could you ever) place your hands flat on the floor without bending your knees?

- Can you now (or could you ever) bend your thumb to touch your forearm?

- As a child, did you amuse your friends by contorting your body into strange shapes or could you do the splits?

- As a child or teenager did your shoulder or kneecap dislocate on more than one occasion?

. Do you consider yourself "double jointed"?

Criterion 2-2 or more of the following (A, B, or C) must be present

Feature A (5 must be present)

. Unusually soft or velvety skin

- Mild skin hyperextensibility

- Unexplained striae distensae or rubae at the back, groins, thighs, breasts, and/or abdomen in adolescence, men or prepubertal

women without a significant gain or loss of body fat or weight

- Bilateral piezogenic papules of the heel

- Recurrent or multiple abdominal hernia(s)

- Atrophic scarring involving at least 2 sites and without the formation of truly papyraceous and/or hemosideric scars as seen in classical EDS. Pelvic floor, rectal, and/or uterine prolapse in children, men, or nulliparous women without a history of morbid obesity or other known predisposing medical condition

- Dental crowding and high or narrow palate

- Arachnodactyly, as defined in one or more of the following: (1) positive wrist sign (Walker sign) on both sides, (2) positive thumb sign (Steinberg sign) on both sides

- Arm span-to-height ratio $>/=1.05$

- Mitral valve prolapse mild or greater based on strict echocardiographic criteria

- Aortic root dilation with $Z$-score $>+2$

Feature B

- Positive family history; 1 or more first-degree relatives independently meeting the current criteria for hEDS

Feature $C$ (must have at least 1)

- Musculoskeletal pain in 2 or more limbs, recurring daily for at least 3 months

- Chronic, widespread pain for $>/=3$ months

. Recurrent joint dislocations or frank joint instability, in the absence of trauma

Criterion 3-all of the following prerequisites MUST be met

- Absence of unusual skin fragility, which should prompt consideration of other types of EDS

- Exclusion of other heritable and acquired connective tissue disorders (CTDs), including autoimmune rheumatologic conditions. In patients with an acquired CTD (eg, lupus, rheumatoid arthritis, etc), additional diagnosis of hEDS requires meeting both features $\mathrm{A}$ and $\mathrm{B}$ of criterion 2 (chronic and/or instability) cannot be counted toward a diagnosis of hEDS in this situation.

- Exclusion of alternative diagnoses that may also include joint hypermobility by means of hypotonia and/or connective tissue laxity. Alternative diagnoses and diagnostic categories include, but are not limited to, neuromuscular disorder (eg, Bethlem myopathy), other hereditary disorders of the connective tissue (eg, other types of EDS, Loeys-Dietz syndrome, Marfan syndrome), and skeletal dysplasias (eg, osteogenesis imperfecta). Exclusion of these considerations may be based upon history, physical examination, and/or molecular genetics testing, as indicated.

Source: https://www.ehlers-danlos.com/wp-content/uploads/hEDS-Dx-Criteria-checklist-1.pdf. 
can remain difficult to clinically differentiate. For example, the confirmation of a positive family history (or lack thereof) may flip the diagnosis from 1 to the other.

The HSDs are defined by joint hypermobility using the Beighton score (Table 2) plus 1 or more secondary musculoskeletal manifestations including post-traumatic symptoms, pain, altered proprioception, and a variety of other characteristics like pes planus, kyphosis, scoliosis, or joint misalignment. ${ }^{6}$ The designation should be used for individuals with symptomatic joint hypermobility when criteria are met and when hEDS has been ruled out. H-HSD is diagnosed in cases of documented prior hypermobility that has disappeared with increased age. ${ }^{7}$

\section{Hypermobility Spectrum Disorders-Incidence and Prevalence}

Accurate characterization of the incidence and prevalence of HSDs and hEDS has been hampered by a lack of its recognition, in part due to the often-benign nature of the condition and the fact that it may improve rather than worsen with aging, as well as by a previously unclear distinction in the literature between HSD and hEDS. Prevalence of generalized joint hypermobility among college-aged students ranges from $12.5 \%$ to $26 \%{ }^{8,9}$ In these college-aged populations, the incidence of hypermobile joints was higher than that in older adults. A more recent UK symptom-based survey estimated prevalence of HSDs in a general population at $3 \% .{ }^{10}$

\section{Genetics in Hypermobility Spectrum Disorders}

HSDs have a strong genetic basis but no known genetic markers. The vast majority of cases do not seem to be linked to any identifiable mutation, although pedigree studies have shown a weak autosomal dominant inheritance pattern with variable penetrance. ${ }^{10,11}$ The EDSs comprise a heterogeneous group of rare monogenic conditions caused by genetic defects in an array of extracellular matrix genes within soft connective tissues. The genetic basis of hEDS remains unknown, in contrast to other well-described EDS subtypes. In view of the considerable clinical overlap with HSDs, many consider HSDs and hEDS to be a single clinical entity, with variable manifestations possibly reflecting variability in penetrance. ${ }^{12}$

\section{The Pathogenesis of Pain in HSDs}

The specific underlying causes and mechanisms of pain in HSD remain poorly understood. Factors likely contributing to the generation and chronicity of pain include nociceptive pain due to structural changes in affected joints, muscle, and connective tissue; neuropathic pain; impaired proprioception and muscle weakness; and central sensitization. These mechanisms are not mutually exclusive and may all contribute to pathogenesis. ${ }^{12}$ Current theories emphasize the importance of localized biomechanical overloading and chronic soft tissue injury due to joint laxity and instability. Repetitive microtrauma may lead to altered kinematics, which, in turn, cause overload on other joints and further soft tissue injury manifesting as arthralgias and diffuse musculoskeletal pain. ${ }^{10,13}$ HSD patients tend to lack proprioceptive acuity, which may increase risk of injury. ${ }^{14}$ In addition, patients tend to have decreased muscle mass and muscle strength, potentially due to increased laxity of tendons, which are unable to transmit power produced by muscles. ${ }^{15,16}$ Altered biomechanics and posture can place overstress on muscles and fascia, causing additional pain.

In addition to these biomechanical abnormalities, patients with HSD may have neurologic disturbances that contribute to diffuse musculoskeletal pain. Generalized hyperalgesia (fibromyalgia) is a common complaint and is believed to be mediated by central sensitization. The biochemical underpinnings of hyperalgesia in HSD are unknown. Some have speculated that it serves an adaptive purpose as a compensatory mechanism to prevent joint instability. ${ }^{17}$ Fear of provoking pain and injury may lead to avoidance of activity, ultimately resulting in deconditioning and exercise intolerance. This often aggravates functional disability and leads to anxiety and depression symptoms, which in turn reduce pain tolerance and promote further pain. ${ }^{16,18}$

\section{Skin Manifestations}

Individuals with HSD and hEDS can display a variety of dermatologic manifestations related to abnormal connective tissue (Table 4). Bruising and bleeding in people with HSD occur due to fragile skin and capillaries rather than abnormal coagulation. ${ }^{19}$

\section{Joint Findings in Patients with HSDs}

Patients present with generalized joint hypermobility with or without joint laxity. While these 2 terms 
Table 4. Skin Manifestations of HSD and hEDS ${ }^{19}$

- Skin hyperextensibility

- Velvety skin texture

- Striae (stretch marks)

- Easy bruising

- Prolonged bleeding (with normal coagulation workup)

- Poor wound healing

- Atrophic-appearing scars (wide, papery, hypopigmented)

are often used interchangeably, laxity indicates instability, whereas hypermobility denotes an increase in the joint's range of motion beyond normal. ${ }^{20}$ Weight-bearing joints are more common sources of pain. Examination may reveal articular or periarticular tenderness, but significant synovitis should prompt reconsideration of the diagnosis and investigation for inflammatory conditions. Small noninflammatory effusions may occur due to repetitive stress on joint structures, and joint deformity may occur and can mimic inflammatory arthritis. Children and young adults may present with scoliosis, pes planus, genu valgum, genu recurvatum, and/or patellar dislocation/subluxation. ${ }^{10}$

People with HSDs often present with a variety of multisystem complaints (Table 5).

\section{HSD in Pregnant Women}

Pregnant women present a unique subset of patients with HSDs in the context of the increased joint laxity normally seen in pregnancy. There is a paucity of research to guide clinicians, but no evidence of increased risks of adverse pregnancy outcomes. ${ }^{22,23}$ There is some elevated risk of late-pregnancy back or sacroiliac pain, along with some risk of early labor, in hEDS and probably in the HSDs. Dysautonomia symptoms often improve as maternal blood volume increases in later pregnancy. Given evidence of an autosomal dominant inheritance pattern with variable penetrance, children of HSD patients may have up to a $50 \%$ chance of hypermobility of some degree.

\section{Affective Disorders (the Neurocognitive Phenotype)}

A positive correlation exists between HSDs and anxiety. Joint hypermobility is more common in patients diagnosed with anxiety, ${ }^{24}$ and anxiety is disproportionately present in people with joint hypermobility. $^{25,26}$

In a study of patients with joint hypermobility, anxiety was found in $70 \%$ of patients in comparison with a $20 \%$ incidence in the age- and gendermatched control group. In the follow-up reverse case-control study, joint hypermobility was found at 17 times the incidence in patients diagnosed with anxiety as compared with age- and gender-matched controls without an anxiety diagnosis. ${ }^{27}$ This correlation between affective state and heritable hypermobility conditions has recently been labeled the "neurocognitive phenotype." Both neuroimaging

Table 5. Multisystem Involvement in Patients with HSD $^{2,10,21}$

\begin{tabular}{|c|c|}
\hline Neurologic & $\begin{array}{l}\text { - Migraine headache } \\
\text { - Tension-type headache } \\
\text { - Dysautonomia } \\
\text { - Postural orthostatic tachycardia syndrome } \\
\text { - Upper limb paresthesias (carpal or cubital tunnel syndromes, thoracic outlet syndrome) }\end{array}$ \\
\hline Gastrointestinal & $\begin{array}{l}\text { - Hiatal hernia } \\
\text { - Gastroesophageal reflux disease } \\
\text { - Irritable bowel syndrome } \\
\text { - Dysphagia } \\
\text { - Constipation } \\
\text { - Crohn's disease }\end{array}$ \\
\hline Psychiatric & $\begin{array}{l}\text { - Anxiety } \\
\text { - Depression } \\
\text { - Insomnia }\end{array}$ \\
\hline Gynecologic & $\begin{array}{l}\text { - Pelvic pain } \\
\text { - Dysmenorrhea } \\
\text { - Urinary stress and/or urge incontinence }\end{array}$ \\
\hline Other & $\begin{array}{l}\text { - Temporomandibular joint disorders } \\
\text { - Chronic fatigue syndrome } \\
\text { - Exercise intolerance } \\
\text { - Postexercise malaise }\end{array}$ \\
\hline
\end{tabular}

HSD, Hypermobility spectrum disorder. 
and genetic studies point to the depth of the interconnectedness of structure and affect.

Eccles and colleagues found that participants with joint hypermobility had significant differences on magnetic resonance imaging (MRI) in brain regions associated with anxiety in comparison to nonhypermobile participants. Hypermobile subjects had significantly enlarged amygdalae bilaterally in comparison to the nonhypermobile cohort. ${ }^{28}$ In addition, the authors found structural differences in the anterior cingulate cortex, another brain structure associated with cognitive control of emotions and a driver of autonomic arousal.

\section{Dysautonomia and Exercise Intolerance}

Problems with autonomic function, such as orthostatic intolerance (ie, postural orthostatic tachycardia syndrome), body temperature dysregulation, and exercise intolerance, are common in people with HSD. ${ }^{29,30}$ While physical activity is often recommended for patients with anxiety, such activity in anxious people with HSD may not have the intended effect. It has been hypothesized that prior unpleasant experiences with physical activity contribute to the kinesiophobia reported in people with joint hypermobility ${ }^{29}$; therefore, like patients with fibromyalgia, people with HSD may not enjoy the anxiolytic benefits of exercise. Coupled with the postexercise malaise that many people with HSD report, a blanket recommendation to increase physical activity may actually exacerbate symptoms. In working with patients who report anxiety, screening for joint hypermobility may offer insights into effectively treating their symptoms of anxiety and in compassionately supporting their desire for wellbeing. Physical activity can be safely initiated even in anxious hypermobile patients if started at low intensity, titrated slowly in a supportive environment, and targeted to the particular biomechanical problems and exercise needs of the individual patient. Support and assistance from behavioral health professionals may be necessary as exercise is introduced.

\section{Challenges in Primary Care}

The primary care provider treating hypermobility syndromes faces many challenges. These may include sorting through multiple and often vague complaints, considering competing diagnoses, determining and often triaging an appropriate workup based on this differential, coordinating a multidisciplinary treatment team, and providing patient education and appropriate self-educational resources once a diagnosis is established.

The patient with HSD will often have a range of complaints involving multiple organ systems. Many of these are not included in the validated questionnaire for hypermobility, and these are often the symptoms that draw the attention of patients and cause them to seek care. These complaints may include manifestations of autonomic dysfunction and other cardiovascular concerns, ${ }^{29}$ a wide range of gastrointestinal issues, ${ }^{21}$ anxiety, phobic disorders, major depression, ${ }^{25}$ headaches and chronic fatigue, ${ }^{10}$ among others. When evaluating a patient with such a wide array of complaints, one may wish to focus on the individual concerns that seem more serious in nature to the provider and those that are most bothersome to the patient.

\section{Differential Diagnosis}

While the initial workup for HSD involves eliminating other potential etiologies, the diagnosis is ultimately clinical and requires no laboratory or imaging tests for confirmation. If a patient presents to the primary care clinic with complaints that suggest HSD, obtaining previous records can prove an important step to avoid a previously completed and costly workup. Using the aptly named "A Simple Questionnaire to Detect Hypermobility" can help streamline an initial evaluation, as it has a sensitivity of $84 \%$ and specificity of $85 \%$ for HSD. ${ }^{1}$ Family history can provide further important information, especially if a first-degree relative has an unequivocal diagnosis of HSD. Identification and/or exclusion of fibromyalgia, complex regional pain syndrome, nerve impingement syndromes, and non-HSD conditions causing hypermobility are important in the evaluation. ${ }^{2}$

HSD and hEDS exist on the same physiologic continuum, but hEDS manifests at the more symptomatic edge of the spectrum. Patients with hEDS generally present to a physician's office at a younger age, have more severe symptoms, and must conform to strict diagnostic criteria put forth in 2017 by the International Consortium on Ehlers-Danlos Syndromes and Related Disorders. ${ }^{3}$ However, once a diagnosis is made, there is no difference in the approach to treatment for HSD and hEDS (Table 6). 


\section{Case 1 Follow-up}

You diagnose Jennifer with Probable hEDS. You get an echocardiogram and an ophthalmologic exam, which are both normal, making a diagnosis of Marfan syndrome unlikely. You discuss possible referral to a genetic counselor with her family, but decide against referral due to the normal exams. The consensus with the family following the meeting is that Jennifer has a presentation consistent with hEDS, for which she meets the 2017 international diagnostic criteria. You refer Jennifer to occupational and physical therapy with an emphasis on postural stabilization and joint protection, avoiding most resistance and isometric exercises. She follows up in 1 month and describes mild improvement in her symptoms. You decide to continue with the current management.

\section{Case 2 Follow-up}

You diagnose Sonja with HSD and refer her to a physical therapist with experience working with HSD patients, to focus on core stability and exercise tolerance.

Additionally, she enters a course of osteopathic manipulative treatment, twice monthly for 3 months, and is restarted on and titrated to a higher dose of duloxetine $(60 \mathrm{mg} / \mathrm{d})$ to address central pain sensitization and improve mood. She is referred for cognitive-behavioral therapy (CBT) to address anxiety and improve coping strategies.

On follow-up 3 months later, she reports significant improvement, with resolution of headaches and brain fog. Her fatigue has improved but not resolved. She continues to suffer from neck and back pain, though with decreased severity and frequency. She feels optimistic that with continued physical therapy, CBT, adequate rest, progressive exercise, and osteopathic treatment, she has found a path forward that promises to empower her to manage her symptoms. She understands that HSD will make her more prone to repetitive stress injury and the importance of maintaining an active lifestyle, including regular stability and core strengthening.

\section{Treatment Options}

Once the diagnosis of HSD or hEDS is suspected or confirmed, the majority of patients can be managed in the primary care setting with a multidisciplinary approach that involves physical therapy, biofeedback, cognitive behavioral therapy/psychotherapy, and pharmacotherapy. ${ }^{31}$ The tenets of pharmacotherapy are similar to those in most chronic pain conditions with avoidance of opioids, preference for nonsteroidal anti-inflammatory drugs (NSAIDs)/ acetaminophen, topical NSAIDs, lidocaine patches where neuropathic pain is present, antidepressants (particularly SNRIs or low-dose tricyclic antidepressants [TCAs]), and/or anticonvulsants. ${ }^{31}$ Symptomatic treatment of muscle pain or spasm may be treated with antispasticity agents (ie, baclofen or tizanidine). Patients with comorbid fibromyalgia may benefit from gabapentin or pregabalin. Patients with exercise intolerance or orthostatic symptoms may not tolerate TCAs or tizanidine.

Successful treatment of chronic pain requires a multidisciplinary approach and should focus on

Table 6. Comparing HSD, hEDS, and Other EDS Disorders

\begin{tabular}{lll}
\hline & HSD & hEDS \\
\hline Joint hypermobility & $\bullet$ & $\bullet$ \\
Chronic pain & $\bullet$ & $\bullet$ \\
Musculoskeletal pain in more than 2 limbs, back $\geq 3$ months & $\bullet$ & $\bullet$ \\
Recurrent joint dislocations & $\bullet$ & $\bullet$ \\
Recurrent hernias, uterine and rectal prolapse & $\bullet$ \\
Marfanoid habitus & $\bullet$ \\
Mild skin hyperextensibility, striae, atrophic scarring & $\bullet$ \\
Skin fragility, pronounced hyperextensibility & $\bullet$ \\
Cardiovascular autonomic dysfunction & $\bullet$ \\
Mild cardiovascular findings (mild mitral valve regurgitation, aortic root dilation) & $\bullet$ \\
Severe cardiovascular findings (aneurysms, arterial rupture) & $\bullet$ \\
Positive family history & $\bullet$ \\
Molecular genetic findings & $\bullet$ \\
\hline
\end{tabular}

HSD, Hypermobility spectrum disorder; hEDS, hypermobile Ehlers-Danlos syndrome; EDS, Ehlers-Danlos syndrome. 
Table 7. Nonpharmacologic Treatments for Hypermobility spectrum disorders (HSDs) ${ }^{10,33-39}$

\begin{tabular}{|c|c|}
\hline Modality & Comments \\
\hline $\begin{array}{l}\text { Joint braces and musculoskeletal } \\
\text { taping }\end{array}$ & May help stabilize joints \\
\hline Biofeedback to improve posture & Improve joint symptoms and proprioception \\
\hline $\begin{array}{l}\text { Osteopathic manipulative } \\
\text { treatment }\end{array}$ & $\begin{array}{l}\text { Reduce joint pain, improve proprioception, increase blood flow. Avoid treatment more than } 3 \\
\text { times per week due to potential for increasing joint laxity. }\end{array}$ \\
\hline Prolotherapy & $\begin{array}{l}\text { Injections in the tendon or ligamentous attachment can trigger a brief inflammatory response } \\
\text { and subsequent reparative cascade to generate new collagen and strengthening the relaxed } \\
\text { connective tissue. May also reduce pain from increased joint stability. }\end{array}$ \\
\hline TENS unit & May be helpful for muscle pain \\
\hline Compressive clothing & $\begin{array}{l}\text { Compression stockings may help combat orthostatic intolerance and tachycardia. Compression } \\
\text { garments may help support loose joints and provide additional proprioceptive input. }\end{array}$ \\
\hline Orthotic shoe inserts & $\begin{array}{l}\text { May improve underlying proprioceptive abnormalities, improve ankle/foot/knee alignment, } \\
\text { reduce joint pain and risk of sprains or other injuries. }\end{array}$ \\
\hline Exercise $^{40}$ & $\begin{array}{l}\text { - Core and joint stabilization. } \\
\text { - Caution with activities that can cause overstretching like yoga or gymnastics. } \\
\text { - Closed kinetic chain exercises (like biking or elliptical trainer). } \\
\text { - Care to avoid overtraining. }\end{array}$ \\
\hline
\end{tabular}

Patient Resources

- Ehlers-Danlos Society (www.ehlers-danlos.com )

- Hypermobility Syndromes Association (www.hypermobility.org)

treating the cause of the pain (eg, dislocation of a joint, muscle imbalance with spasm, sprain/strain), reducing pain where possible, and maximizing functional capacity and quality of life. It should also be noted that fatigue and pain seem to be linked, leading to decreased quality of life. Treating dysautonomia in patients with HSD may improve both fatigue and pain. ${ }^{32}$

Lifestyle modifications, regular exercise without overtraining, and stretching techniques focused on tight muscles (but not lax joints) may help. Physical and occupational therapy programs centered on strengthening the core and periarticular musculature can improve proprioception, reduce injury, and reduce pain while improving function (see Table 7).

\section{Conclusion}

Primary care clinicians will frequently see patients with hypermobility syndrome disorders. Recognition of the diagnostic criteria, common overlaps, and differentiating features of the various disorders under this umbrella will help clinicians appropriately diagnose patients and develop multidisciplinary treatment approaches to optimize outcomes.

\section{References}

1. Hakim AJ, Grahame R. A simple questionnaire to detect hypermobility: an adjunct to the assessment of patients with diffuse musculoskeletal pain. Int J Clin Pract 2003;57:163-6.

2. Fikree A, Aziz Q, Grahame R. Joint hypermobility syndrome. Rheum Dis Clin North Am 2013;39:419-30.

3. 2017 International Diagnostic Criteria for hEDS. 2021. Available from: https://ehlers-danlos.com/ wp-content/uploads/hEDS-Dx-Criteria-checklist1.pdf.

4. Kirk JA, Ansell BM, Bywaters EG. The hypermobility syndrome: musculoskeletal complaints associated with generalized joint hypermobility. Ann Rheum Dis 1967;26:419-25.

5. Malfait F, Francomano C, Byers P. The 2017 international classification of the Ehlers-Danlos syndromes. Am J Med Genet C Semin Med Genet 2017;175:8-26.

6. What is HSD. 2021. Available from: https://www. ehlers-danlos.com/what-is-hsd/.

7. Castori M, Tinkle B, Levy H, Grahame R, Malfait F, Hakim A. A framework for the classification of joint hypermobility and related conditions. Am J Med Genet Part C Semin Med Genet 2017; $175 ; 148-57$.

8. Russek LN, Errico DM. Prevalence, injury rate and symptom frequency in generalized joint laxity and joint hypermobility syndrome in a "healthy" college population. Clin Rheumatol 2016;35:1029-39. 
9. Reuter PR, Fichthorn KR. Prevalence of generalized joint hypermobility, musculoskeletal injuries, and chronic musculoskeletal pain among American university students. Peer J 2019;7:e7625.

10. Kumar B, Lenert P. Joint hypermobility syndrome: recognizing a commonly overlooked cause of chronic pain. Am J Med 2017;130:640-7.

11. Castori M, Sperduti I, Celletti C, Camerota F, Grammatico P. Symptom and joint mobility progression in the joint hypermobility syndrome (EhlersDanlos syndrome, hypermobility type). Clin Exp Rheumatol 2011;29:998-1005.

12. Syx D, Wandele I, Rombaut L, Malfait F. Hypermobility, the Ehlers-Danlos syndromes and chronic pain. Clin Exp Rheumatol 2017;35:S116-S122.

13. Castori M, Morlino S, Celletti C, et al. Re-writing the natural history of pain and related symptoms in the joint hypermobility syndrome/Ehlers-Danlos syndrome, hypermobility type. Am J Med Genet A 2013;161A:2989-3004.

14. Bates AV, Alexander CM. Kinematics and kinetics of people who are hypermobile: a systematic review. Gait Posture 2015;41:361-9.

15. Pacey V, Nicholson LL, Adams RD, et al. Generalized joint hypermobility and risk of lower limb joint injury during sport: a systematic review with meta-analysis. Am J Sports Med 2010;38:1487-97.

16. Rombaut L, Malfait F, De Wandele I, et al. Muscle mass, muscle strength, functional performance, and physical impairment in women with the hypermobility type of Ehlers-Danlos syndrome. Arthritis Care Res (Hoboken) 2012;64:1584-92.

17. Rombaut L, Scheper M, De Wandele I, et al. Chronic pain in patients with the hypermobility type of Ehlers-Danlos syndrome: evidence for generalized hyperalgesia. Clin Rheumatol 2015;34:1121-9.

18. Scheper M, de Vries J, Verbunt J, et al. Chronic pain in hypermobility syndrome and Ehlers-Danlos syndrome (hypermobility type): it is a challenge. J Pain Res 2015;8:591-601.

19. Hakim AJ, Sahota A. Joint hypermobility and skin elasticity: the hereditary disorders of connective tissue. Clin Dermatol 2006;24:521-33.

20. Tinkle BT, Levy HP. Symptomatic joint hypermobility: the hypermobile type of Ehlers-Danlos syndrome and the hypermobility spectrum disorders. Med Clin North Am 2019;103:1021-33.

21. Beckers AB, Keszthelyi D, Fikree A, et al. Gastrointestinal disorders in joint hypermobility syndrome/Ehlers-Danlos syndrome hypermobility type: a review for the gastroenterologist. Neurogastroenterol Motil 2017;29:e13013.

22. Sundelin HE, Stephansson O, Johansson K, Ludvigsson JF. Pregnancy outcome in joint hypermobility syndrome and Ehlers-Danlos syndrome. Acta Obstet Gynecol Scand 2017;96:114-9.
23. Karthikeyan A, Venkat-Raman N. Hypermobile Ehlers-Danlos syndrome and pregnancy. Obstet Med 2018;11:104-9.

24. Bulbena A, Duro JC, Mateo A, Porta M, Vallejo J. Joint hypermobility syndrome and anxiety disorders. Lancet 1988;2:694.

25. Bulbena A, Duro JC, Porta M, et al. Anxiety disorders in the joint hypermobility syndrome. Psychiatry Res 1993;46:59-68.

26. Martín-Santos R, Bulbena A, Porta M, Gago J, Molina L, Duró JC. Association between joint hypermobility syndrome and panic disorder. AJP 1998;155:1578-83.

27. Pailhez G, Castano J, Rosado S, et al. 2015. Joint hypermobility, anxiety, and psychosomatics - the new neuroconnective phenotype. Available from: https://www.intechopen.com/books/a-fresh-lookat-anxiety-disorders/joint-hypermobility-anxiety-andpsychosomatics-the-new-neuroconnective-phenotype.

28. Eccles JA, Beacher FDC, Gray MA, Jones CL, et al. Brain structure and joint hypermobility: relevance to the expression of psychiatric symptoms. $\mathrm{Br} \mathrm{J}$ Psychiatry 2012;200:508-9.

29. Gazit Y, Nahir AM, Grahame R, Jacob G. Dysautonomia in the joint hypermobility syndrome. Am J Med 2003;115:33-40.

30. Hakim A, O'Callaghan C, De Wandele I, Stiles L, Pocinki A, Rowe P. Cardiovascular autonomic dysfunction in Ehlers-Danlos syndrome-hypermobile type. Am J Med Genet C Semin Med Genet 2017;175:168-74.

31. Chora P, Tinkle B, Hamonet C, et al. Pain management in the Ehlers-Danlos syndromes. Am J Med Genet Part C Semin Med Genet 2017;9999C:1-8.

32. Bravo J, Sanhueza FG, Hakim AJ. Cardiovascular autonomic dysfunction and chronic fatigue in fibromyalgia and joint hypermobility syndrome. In: Hypermobility, fibromyalgia and chronic pain. London: Churchill Livingstone; 2010. p. 69-82.

33. Simmonds JV, Keer RJ. Hypermobility and the hypermobility syndrome, part 2: assessment and management of hypermobility syndrome: illustrated via case studies. Man Ther 2008;13:e1-e11.

34. Simpson M. Benign joint hypermobility syndrome: evaluation, diagnosis, and management. J Am Osteopath Assoc 2006;106:531-63.

35. Hauser RA, Phillips HJ. Treatment of joint hypermobility syndrome, including Ehlers-Danlos syndrome, with Hackett-Hemwall prolotherapy. Journal of Prolo 2011;3:612-29.

36. Hamonet C, Laouar R, Vienne M, Brissot R, Bernard J-C, Comberg A. $V^{\wedge}$ etements compressifs et syndrome d'Ehlers-Danlos. Etude multicentrique et prospective sur 49 personnes du handicap avec le Handitest. Journal de Readaptation Medicale 2010;30:184-91. 
37. Hamonet C, Brock I. Joint mobility and EhlersDanlos syndrome (EDS): new data based on 232 cases. J Arthritis 2015;4:148.

38. Ferrell WR, Tennant N, Sturrock RD, et al. Amelioration of symptoms by enhancement of proprioception in patients with joint hypermobility syndrome. Arthritis Rheum 2004;50:3323-8.
39. Chopra P, Tinkle B, Hamonet C, et al. Pain management in the Ehlers-Danlos syndromes. Am J Med Genet C Semin Med Genet 2017;175:212-9.

40. Ferrell WR, Tennant N, Sturrock RD, et al Amelioration of symptoms by enhancement of proprioception in patients with joint hypermobility syndromes. Arthritis Rheumatism 2004;50:3323-8. 